\title{
Factors Affecting Postoperative Complications and Out- comes of Cervical Spondylotic Myelopathy with Cerebral Palsy : A Retrospective Analysis
}

\author{
Hyung Cheol Kim, ${ }^{1, *}$ Hyeongseok Jeon, ${ }^{1,+}$ Yeong Ha Jeong, ${ }^{1}$ Sangman Park, ${ }^{1}$ Seong Bae An, ${ }^{1, \pm}$ Jeong Hyun Heo, ${ }^{2,3}$ \\ Dong Ah Shin, ${ }^{1}$ Seong Yi, Keung Nyun Kim, ${ }^{1}$ Yoon Ha, ${ }^{1}$ Sung-Rae Cho ${ }^{2,3,4,5}$ \\ Department of Neurosurgery,' Spine and Spinal Cord Institute, Severance Hospital, Yonsei University College of Medicine, Seoul, Korea \\ Department and Research Institute of Rehabilitation Medicine, ${ }^{2}$ Severance Hospital, Yonsei University College of Medicine, Seoul, Korea \\ Graduate Program of NanoScience and Technology, ${ }^{3}$ Yonsei University College of Medicine, Seoul, Korea \\ Brain Korea 21 PLUS Project for Medical Science, ${ }^{4}$ Yonsei University College of Medicine, Seoul, Korea \\ Rehabilitation Institute of Neuromuscular Disease, ${ }^{5}$ Yonsei University College of Medicine, Seoul, Korea
}

Objective : Cervical surgery in patients with cervical spondylotic myelopathy (CSM) and cerebral palsy (CP) is challenging owing to the complexities of the deformity. We assessed factors affecting postoperative complications and outcomes after CSM surgery in patients with CP.

Methods : Thirty-five consecutive patients with CP and CSM who underwent cervical operations between January 2006 and January 2014 were matched to 35 non-cerebral palsy (NCP) control patients. Postoperative complications and radiologic outcomes were compared between the groups. In the CP group, the Japanese Orthopaedic Association score; Oswestry neck disability index; modified Barthel index; and values for the grip and pinch, Box and Block, and Jebsen-Taylor hand function tests were obtained preand postoperatively and compared between those with and without postoperative complications.

Results : Sixteen patients (16/35\%) in the CP group and seven $(7 / 35 \%)$ in the NCP group ( $p=0.021)$ had postoperative complications. Adjacent segment degeneration ( $p=0.021)$, postoperative motor weakness $(p=0.037)$, and revisions $(p=0.003)$ were significantly more frequent in the CP group than in the NCP group; however, instrument-related complications were not significantly higher in the $\mathrm{CP}$ group $(7 / 35$ vs. $5 / 35, p=0.280)$. The number of preoperative fixed cervical deformities were significantly higher in $\mathrm{CP}$ with postoperative complications (5/16 vs. 1/19, $p=0.037$ ). In the CP group, clinical outcomes were almost similar between those with and without postoperative complications.

Conclusion : The occurrence of complications during the follow-up period was high in patients with CP. However, postoperative complications did not significantly affect clinical outcomes.

Key Words : Cervical spondylotic myelopathy · Cerebral palsy · Postoperative complications · Kyhposis · Pseudoarthrosis.

- Received : January 13, 2021 • Revised : February 28, 2021 •Accepted : March 10, 2021

- Address for reprints : Yoon Ha

Department of Neurosurgery, Spine and Spinal Cord Institute, Severance Hospital, Yonsei University College of Medicine, 50-1 Yonsei-ro, Seodaemun-gu, Seoul 03722, Korea Tel : +82-2-2228-2165, Fax : +82-2-393-9979, E-mail : hayoon@yuhs.ac, ORCID : https://orcid.org/0000-0002-3775-2324

\section{Sung-Rae Cho}

Department and Research Institute of Rehabilitation Medicine, Severance Hospital, Yonsei University College of Medicine, 50-1 Yonsei-ro, Seodaemun-gu, Seoul 03722, Korea Tel : +82-2-2228-3715, Fax : +82-2-363-2795, E-mail : srch0918@yuhs.ac, ORCID : https://orcid.org/0000-0003-1429-2684

${ }^{*}$ Current affiliation : Department of Neurosurgery, Bundang Jesaeng General Hospital, Seongnam, Korea; ${ }^{\dagger}$ Current affiliation : Knee and Spine Hospital, Seoul, Korea; ${ }^{\ddagger}$ Current affiliation : Department of Neurosurgery, Spine Center, CHA Bundang Medical Center, CHA University School of Medicine, Seongnam, Korea 


\section{INTRODUCTION}

Patients with cerebral palsy (CP) demonstrate involuntary and repetitive neck movements and are more likely to develop cervical spondylotic myelopathy (CSM $)^{1,5,6,10,18)}$. Because CSM progresses in the 30-40-year age range, individuals with CP often develop various neurological defects later in life. The primary pathologic factors that lead to serious disability include compression against neural elements caused by canal stenosis from excessive spondylotic changes and severe dynamic instability of the spine induced by sustained involuntary movements and malalignment of the cervical spine ${ }^{16)}$. Typically, conservative treatments are ineffective; patients often require surgical treatment ${ }^{2}$. The most important surgical objectives for such patients are adequate decompression of the spinal cord and nerve roots, stabilization of the cervical spine, and alignment correction $^{11,12,15)}$. Recent advances in medical technology have led to the development of improved internal fixation methods, which promote stronger initial mechanical stability resulting from anterior plating or posterior screw fixation ${ }^{7)}$. CSM surgery remains challenging, owing to the complexity of the deformi$\mathrm{ty}^{8,20,27,28)}$. Additionally, involuntary neck movements after surgical treatment can worsen neurological symptoms or further complications associated with surgical implants, frequently leading to poor long-term prognoses ${ }^{12)}$.

We retrospectively investigated the prevalence of various postoperative complications and outcomes resulting from CSM surgery in patients with CP and compared the results with those of non-cerebral palsy (NCP) patients with similar surgical treatments.

\section{MATERIALS AND METHODS}

All procedures were performed in accordance with the ethical standards of the Institutional Review Board of Severance Hospital, Yonsei University College of Medicine (approval number : 2016-1447-001). The requirement for informed consent was waived because of the retrospective design of this study.

\section{Patients}

Between January 2006 and January 2014, we retrospectively identified 77 patients with CP diagnosed in the Department of Rehabilitation Medicine and Neurosurgery and CSM who underwent surgeries using cervical anterior (anterior cervical discectomy and fusion or anterior cervical corpectomy and fusion), posterior (posterior decompressive laminectomy and lateral mass screw or pedicle screw fixation), or combined (anterior and posterior) approach in our institute due to intractable pain or progressive weakness. The surgical method and implantation levels were determined by evaluating the extent of the correlation between radiological findings and clinical symptoms. Inclusion criteria were as follows : age $>18$ years with CP and CSM; subaxial cervical spine lesions; intractable pain or progressive weakness and baseline modified Japanese Orthopaedic Association (mJOA) score $\leq 18$; follow-up period $>2$ years; and complete medical record of clinical and radiologic outcomes in the follow-up period. Exclusion criteria were as follows : infections, autoimmune state, tumors, or other pathologic conditions; follow-up period $<2$ years; and lack of clinical and radiologic outcome data because of poor image quality or follow-up loss. We excluded 42 patients with a follow-up period $<2$ years or missing clinical and radiologic outcome data. A total of 35 consecutive patients with CP and CSM who underwent cervical surgeries were ultimately included. Regardless of the type of CP before surgery, patients who did not have complications wore the postoperative orthosis for up to 3 months. Patients with complications that were found within 3 months postoperatively wore the orthosis for an additional 2-3 months depending on their condition.

Patient demographics included cervical spine alignment (C0-2 and C2-7 Cobb angle, C2-7 sagittal vertical axis [SVA], chin brow vertical angle [CBVA], and T1 slope [T1S] minus the $\mathrm{C} 2-7$ Cobb angle) and postoperative complications. We paired the participants with 35 age-, sex-, and operation levelmatched NCP patients who also underwent cervical surgeries owing to CSM.

\section{Data collection}

Relevant patient data, including disease history, physical examination findings, and radiological outcomes, were obtained from medical records.

\section{Radiologic outcomes}

Plain radiographs, including anterior-posterior and lateral views, were obtained before and 2 years postoperatively, to assess cervical alignment. The $\mathrm{C} 0-2$ Cobb angle (neutral posi- 
tion), C2-7 Cobb angle (cervical lordosis, CL) (neutral, flexion, and extension positions), T1S, C2-7 SVA, and CBVA were determined. The $\mathrm{C} 0-2 \mathrm{Cobb}$ angle was measured as the angle between the McGregor line and lower endplate of the $\mathrm{C} 2$ vertebra (positive values indicate lordosis and negative values indicate kyphosis between the occiput and C2). CL was defined as the angle between two crossed perpendicular lines extending parallel to the inferior endplate of $\mathrm{C} 2$ and $\mathrm{C} 7$ on a standing lateral radiograph of the cervical spine. The C2-7 SVA was defined as the distance between a plumb line dropped from the center of C2 (or dens) and the posterosuperior aspect of C7. The T1S was measured as the angle between a horizontal plane and a line parallel to the superior T1 endplate. We also evaluated T1S minus CL (T1S-CL), which is a cervical analog to the pelvic incidence minus lumbar lordosis mismatch ${ }^{14)}$. The C2-7 range of motion (ROM) was calculated by subtracting the CL on flexion from that on extension. The CBVA was defined as the angle between a line from the brow to the chin to the vertical.

\section{Postoperative complications}

Postoperative complications, including neurologic deteriorations, adjacent segment disease, instrument-related complications, and revision frequency, were recorded until the last follow-up (at least 2 years after surgery). We also investigated each complication according to the follow-up period.

\section{Clinical outcomes}

All clinical outcome assessments, including the mJOA score (range, $0-21 ; 0=$ maximal neurological deficits, $21=$ no neurological deficits) ${ }^{34)}$, Oswestry neck disability index (NDI) questionnaire (10 questions addressing pain intensity ${ }^{22)}$, personal care, lifting, reading, headaches, concentration, working, driving, sleeping, and recreation, with higher scores indicating worse outcomes), and the modified Barthel index (MBI; range, 0-100; higher scores indicate greater functional independence), were performed preoperatively and 24 months postoperatively in the CP group. Clinical outcomes were also evaluated by a rehabilitation physician using the following tests : grip and pinch (grasping power), Box and Block (performance while carrying a box and block for 1 minute), and Jebsen-Taylor hand function (time to perform certain tasks, including writing, turning cards, picking up small objects, eating, stacking checkers, and carrying light/heavy cans).

\section{Statistical analysis}

Data are reported as means \pm standard deviations. Group differences in demographic and radiologic parameters were evaluated using the chi-square or Fisher's exact test, as appropriate. The paired t-test was used to evaluate differences in clinical parameters between before and 2 years after surgery. Statistical analyses were performed using IBM SPSS Statistics for Windows, version 19 (IBM Corp., Armonk, NY, USA). $p<0.05$ was considered significant.

\section{RESULTS}

\section{Patient demographics}

The demographic and clinical characteristics of the patients (35 in each group) are presented in Table 1. In the CP group, there were 21 patients with dyskinetic $C P$, five with spastic $C P$, and nine with mixed CP. The average height $(p=0.006)$, weight $(p<0.001)$, body mass index $(p=0.001)$, and bone mineral density $(p<0.001)$ were significantly lower, and the mean followup duration was significantly longer $(p=0.001)$ in the CP group than in the NCP group. Osteoporosis $(p=0.007)$ and fixed cervical kyphosis $(p=0.003)$ were significantly more frequent in the CP group than in the NCP group. There were no significant differences in age $(p=0.610)$, sex ratio $(p=1.000)$, comorbidities (hypertension, $p=0.690$; diabetes, $p=0.551$ ), and surgical approach $(p=0.075)$ between the groups.

\section{Radiologic outcomes}

Preoperative and 2-year postoperative cervical sagittal alignment parameters are summarized according to groups in Table 2. During the preoperative period, the CL on flexion was significantly lower in the CP group than in the NCP group ( $p=0.008)$. The T1S $(p=0.035)$ and C $2-7 \mathrm{ROM}(p=0.011)$ were significantly higher in the CP group than in the NCP group; however, the C2-7 SVA ( $p=0.245)$, CBVA ( $p=0.893)$, and T1S minus the C2-C7 Cobb angle $(p=0.180)$ did not differ significantly. In the postoperative period, the C2-7 Cobb angle on flexion was the only parameter that significantly differed between the groups $(p=0.019)$. There was no significant difference in radiologic parameters between the groups before and after surgery. 
Table 1. Baseline characteristics

\begin{tabular}{|c|c|c|c|}
\hline & $\mathrm{CP}(n=35)$ & $\operatorname{NCP}(n=35)$ & $p$-value \\
\hline \multicolumn{4}{|l|}{ Type of CP } \\
\hline Dyskinetic, athetoid & 21 & & \\
\hline Spastic, diplegic & 5 & & \\
\hline Mixed & 9 & & \\
\hline \multicolumn{4}{|l|}{ Demographic data } \\
\hline Age (years) & $40.0 \pm 9.6$ & $41.2 \pm 9.6$ & 0.610 \\
\hline Sex, male & 21 & 21 & 1.000 \\
\hline Height (cm) & $160.8 \pm 8.6$ & $167.0 \pm 9.5$ & $0.006^{*}$ \\
\hline Weight (kg) & $53.8 \pm 12.1$ & $67.5 \pm 11.8$ & $<0.001^{*}$ \\
\hline $\mathrm{BMI}\left(\mathrm{kg} / \mathrm{m}^{2}\right)$ & $20.8 \pm 4.5$ & $24.1 \pm 2.9$ & $0.001^{*}$ \\
\hline BMD & $-1.2 \pm 1.1$ & $0.4 \pm 1.0$ & $<0.001^{*}$ \\
\hline $\begin{array}{l}\text { Osteoporosis, T-score } \\
<-2.5\end{array}$ & 5 & 0 & $0.007^{*}$ \\
\hline HTN & 3 & 4 & 0.690 \\
\hline DM & 1 & 2 & 0.551 \\
\hline $\begin{array}{l}\text { Follow up duration } \\
\text { (months) }\end{array}$ & 57.7 & 32.3 & $0.001^{*}$ \\
\hline $\begin{array}{l}\text { Fixed cervical } \\
\text { kyphosis }\end{array}$ & 6 & 0 & $0.003^{*}$ \\
\hline Approach of surgery & & & 0.075 \\
\hline Ant. approach only & 20 & 27 & - \\
\hline $\begin{array}{l}\text { Post. with/without } \\
\text { ant. approach }\end{array}$ & 15 & 8 & - \\
\hline
\end{tabular}

Values are presented as mean \pm standard deviation or number. ${ }^{*}$ Indicates statistical significance $(p<0.05)$. CP : cerebral palsy, NCP : non-cerebral palsy, BMI : body mass index, BMD : bone mineral density, HTN : hypertension, DM : diabetes mellitus, Ant. : anterior, Post. : posterior

\section{Postoperative complications}

Postoperative complications are summarized in Table 3. There were 16 patients $(45.7 \%)$ with postoperative complications in the CP group and seven (20.0\%) in the NCP group $(p=0.021)$. Although the number of overall neurologic deterioration events did not significantly differ between the groups ( $p=0.324$ ), motor weakness was significantly more frequent in the CP group than in the NCP group $(p=0.037)$. The groups did not significantly differ in overall instrument-related complications, including pseudoarthrosis, screw loosening, and screw fracture $(p=0.280)$; however, adjacent segment degeneration (ASD; $p=0.018)$ and revision events $(p=0.003)$ were significantly more frequent in the $\mathrm{CP}$ group than in the NCP group. Among those with postoperative complications, only
Table 2. Radiologic outcomes between the CP group and the NCP group

\begin{tabular}{|c|c|c|c|}
\hline & $\begin{array}{c}\mathrm{CP} \\
(n=35)\end{array}$ & $\begin{array}{l}\text { NCP } \\
(n=35)\end{array}$ & $p$-value \\
\hline \multicolumn{4}{|l|}{ Pre-op radiologic parameters } \\
\hline CO-C2 angle, neutral $\left(^{\circ}\right)$ & $40.6 \pm 17.0$ & $37.8 \pm 7.8$ & 0.370 \\
\hline C2-C7 angle, neutral $\left(^{\circ}\right)$ & $4.3 \pm 24.2$ & $3.0 \pm 8.5$ & 0.778 \\
\hline C2-C7 angle, flexion $\left(^{\circ}\right)$ & $-31.0 \pm 17.3$ & $-19.4 \pm 18.1$ & $0.008^{*}$ \\
\hline C2-C7 angle, extension $\left({ }^{\circ}\right)$ & $22.3 \pm 21.6$ & $18.6 \pm 12.6$ & 0.380 \\
\hline T1 slope $\left(^{\circ}\right)$ & $26.4 \pm 13.8$ & $20.9 \pm 5.9$ & $0.035^{*}$ \\
\hline T1 slope-C27 angle $\left({ }^{\circ}\right)$ & $22.1 \pm 17.3$ & $17.9 \pm 6.3$ & 0.180 \\
\hline C2-7 SVA (mm) & $26.0 \pm 22.7$ & $21.4 \pm 6.6$ & 0.245 \\
\hline C2-7 ROM $\left(^{\circ}\right)$ & $53.3 \pm 24.3$ & $38.0 \pm 24.4$ & $0.011^{*}$ \\
\hline $\operatorname{CBVA}\left({ }^{\circ}\right)$ & $-1.8 \pm 16.1$ & $-1.5 \pm 5.6$ & 0.893 \\
\hline \multicolumn{4}{|l|}{ Post-op radiologic parameters } \\
\hline C0-C2 angle, neutral $\left(^{\circ}\right)$ & $40.7 \pm 19.8$ & $40.7 \pm 7.6$ & 0.992 \\
\hline C2-C7 angle, neutral $\left(^{\circ}\right)$ & $2.2 \pm 23.3$ & $5.5 \pm 10.7$ & 0.459 \\
\hline C2-C7 angle, flexion $\left(^{\circ}\right)$ & $-23.3 \pm 15.9$ & $-15.2 \pm 12.1$ & $0.019^{*}$ \\
\hline C2-C7 angle, extension $\left({ }^{\circ}\right)$ & $13.6 \pm 23.0$ & $18.4 \pm 10.3$ & 0.270 \\
\hline T1 slope $\left(^{\circ}\right)$ & $24.7 \pm 13.4$ & $23.8 \pm 5.7$ & 0.702 \\
\hline T1 slope-C27 angle $\left(^{\circ}\right)$ & $22.5 \pm 18.3$ & $18.0 \pm 9.6$ & 0.204 \\
\hline C2-7 SVA (mm) & $25.6 \pm 24.0$ & $18.0 \pm 8.9$ & 0.081 \\
\hline C2-7 ROM $\left(^{\circ}\right)$ & $36.9 \pm 27.3$ & $33.5 \pm 16.3$ & 0.535 \\
\hline $\operatorname{CBVA}\left({ }^{\circ}\right)$ & $-1.3 \pm 11.7$ & $-1.9 \pm 6.5$ & 0.790 \\
\hline \multicolumn{4}{|c|}{$\begin{array}{l}\text { Variation between pre- and postop } \\
\text { radiologic parameters }\end{array}$} \\
\hline C0-C2 angle, neutral $\left({ }^{\circ}\right)$ & $0.1 \pm 23.3$ & $2.9 \pm 5.9$ & 0.490 \\
\hline C2-C7 angle, neutral $\left(^{\circ}\right)$ & $-2.0 \pm 18.9$ & $2.4 \pm 12.1$ & 0.245 \\
\hline C2-C7 angle, flexion $\left(^{\circ}\right)$ & $7.7 \pm 19.2$ & $4.3 \pm 17.2$ & 0.428 \\
\hline C2-C7 angle, extension $\left({ }^{\circ}\right)$ & $-8.7 \pm 23.5$ & $-0.2 \pm 13.0$ & 0.067 \\
\hline T1 slope $\left(^{\circ}\right)$ & $-1.6 \pm 11.0$ & $2.9 \pm 7.8$ & 0.052 \\
\hline T1 slope-C27 angle $\left(^{\circ}\right)$ & $0.4 \pm 18.6$ & $0.1 \pm 10.4$ & 0.942 \\
\hline C2-7 SVA (mm) & $-0.4 \pm 21.7$ & $-3.4 \pm 11.1$ & 0.471 \\
\hline C2-7 ROM $\left(^{\circ}\right)$ & $-16.4 \pm 32.6$ & $-4.5 \pm 24.1$ & 0.086 \\
\hline $\operatorname{CBVA}\left({ }^{\circ}\right)$ & $0.6 \pm 18.5$ & $-0.4 \pm 8.4$ & 0.774 \\
\hline
\end{tabular}

Values are presented as mean \pm standard deviation. ${ }^{*}$ Indicates statistical significance $(p<0.05)$. CP : cerebral palsy, NCP : non-cerebral palsy, Preop : preoperative, SVA : sagittal vertical axis, ROM : range of motion, CBVA : chin brow vertical angle, Post-op : postoperative

the incidence of neurological deterioration significantly differed between groups ( $p=0.027$ ).

In addition, the occurrence of neurologic deterioration was significantly high in the CP group between 2 and 5 years after 
Table 3. Distribution of postoperative complications

\begin{tabular}{|c|c|c|c|}
\hline & $\underset{(n=35)}{C P}$ & $\begin{array}{c}\text { NCP } \\
(n=35)\end{array}$ & $p$-value \\
\hline Overall complications & $16(45.7)$ & $7(20.0)$ & $0.021^{*}$ \\
\hline Neurologic deteriorations & $7(20.0)$ & $4(11.5)$ & 0.324 \\
\hline $\begin{array}{l}\text { Pain, sensory change without } \\
\text { weakness }\end{array}$ & $1(2.8)$ & $3(8.5)$ & 0.293 \\
\hline Motor weakness & $6(17.1)$ & $1(2.8)$ & $0.037^{*}$ \\
\hline ASD & $7(20.0)$ & $1(2.8)$ & $0.018^{*}$ \\
\hline Instruments related complications & $7(20.0)$ & $5(14.3)$ & 0.280 \\
\hline Pseudoarthrosis, non-fusion & $2(5.6)$ & $2(5.6)$ & 1.000 \\
\hline Screw loosening or fracture & $5(14.3)$ & $3(8.5)$ & 0.450 \\
\hline Revision & $6(17.1)$ & $0(0.0)$ & $0.003^{*}$ \\
\hline ASD & $3(8.5)$ & $0(0.0)$ & - \\
\hline Motor weakness & $6(17.1)$ & $1(2.8)$ & - \\
\hline Instrument related complications & $1(2.8)$ & $0(0.0)$ & - \\
\hline \multicolumn{4}{|l|}{ Period of complications } \\
\hline$\leq$ Post-op 1 year & 35 & 35 & \\
\hline Overall complications & $5(14.3)$ & $5(14.3)$ & 1.000 \\
\hline ASD & $0(0.0)$ & $0(0.0)$ & - \\
\hline Neurologic deteriorations & $1(2.8)$ & $3(8.6)$ & 0.293 \\
\hline Instruments related complications & $4(11.4)$ & $3(9.6)$ & 0.690 \\
\hline Revision & $1(2.8)$ & $0(0.0)$ & 0.317 \\
\hline Post-op 1 year to post-op 2 years & 35 & 35 & \\
\hline Overall complications & $3(8.6)$ & $1(2.8)$ & 0.293 \\
\hline ASD & $1(2.8)$ & $0(0.0)$ & 0.237 \\
\hline Neurologic deteriorations & $1(2.8)$ & $0(0.0)$ & 0.237 \\
\hline Instruments related complications & $2(5.6)$ & $1(2.8)$ & 0.551 \\
\hline Revision & $1(2.8)$ & $0(0.0)$ & 0.237 \\
\hline Post-op 2 years to post-op 5 years & 35 & 16 & \\
\hline Overall complications & $8(22.9)$ & $3(18.8)$ & 0.738 \\
\hline ASD & $3(8.6)$ & $3(18.8)$ & 0.311 \\
\hline Neurologic deteriorations & $6(17.1)$ & $0(0.0)$ & $0.027^{*}$ \\
\hline Instruments related complications & $2(5.6)$ & $0(0.0)$ & 0.214 \\
\hline Revision & $3(8.6)$ & $0(0.0)$ & 0.126 \\
\hline$>$ Post-op 5 years & 16 & 7 & \\
\hline Overall complications & $4(25.0)$ & $0(0.0)$ & 0.071 \\
\hline ASD & $4(25.0)$ & $2(28.6)$ & 0.858 \\
\hline Neurologic deteriorations & $2(12.5)$ & $0(0.0)$ & 0.216 \\
\hline Instruments related complications & $0(0.0)$ & $0(0.0)$ & - \\
\hline Revision & $2(12.5)$ & $0(0.0)$ & 0.216 \\
\hline
\end{tabular}

Values are presented as number (\%). ${ }^{*}$ Indicates statistical significance $(p<0.05)$. CP : cerebral palsy, NCP : non-cerebral palsy, ASD : adjacent segment disease, Post-op : postoperative
Table 4. Baseline characteristics according to the presence or absence of complications in the cerebral palsy group

\begin{tabular}{lccc} 
& YC $(\mathbf{n}=\mathbf{1 6})$ & NC $(\mathbf{n}=\mathbf{1 9})$ & $\boldsymbol{p}$-value \\
\hline Age (years) & $37.95 \pm 7.06$ & $42.80 \pm 11.83$ & 0.140 \\
Sex, male & 12 & 9 & 0.096 \\
\hline Height $(\mathrm{cm})$ & $159.8 \pm 7.4$ & $162.1 \pm 10.1$ & 0.447 \\
Weight $(\mathrm{Kg})$ & $55.5 \pm 14.3$ & $51.5 \pm 8.3$ & 0.337 \\
BMI $\left(\mathrm{Kg} / \mathrm{m}^{2}\right)$ & $21.7 \pm 5.3$ & $19.6 \pm 2.7$ & 0.176 \\
BMD & $-1.43 \pm 1.01$ & $-1.10 \pm 1.13$ & 0.386 \\
Osteoporosis & 2 & 3 & 0.781 \\
HTN & 1 & 2 & 0.649 \\
\hline DM & 0 & 1 & 0.264 \\
Type of CP & & & 0.785 \\
Dyskinetic, athetoid & 9 & 12 & \\
Spastic, diplegic & 3 & 2 & \\
Mixed & 4 & 5 & \\
Preoperative history & 1 & 0 & 0.206 \\
Fixed cervical deformity & 5 & 1 & $0.037^{*}$ \\
\hline Perioperative Botox injection & 12 & 16 & 0.498 \\
\hline
\end{tabular}

Values are presented as mean \pm standard deviation or number. ${ }^{*}$ Indicates statistical significance $(p<0.05)$. YC : yes complication, NC : no complication, $\mathrm{BMI}$ : body mass index, BMD : bone mineral density, HTN : hypertension, $\mathrm{DM}$ : diabetes mellitus, $\mathrm{CP}$ : cerebral palsy

surgery $(p=0.027)$.

\section{Clinical and radiologic outcomes in the $\mathrm{CP}$ group}

A summary of demographics and clinical outcomes in the $\mathrm{CP}$ group is clustered and presented according to the presence or absence of complications in Tables 4 and 5. In the complication group, the number of fixed cervical deformity was significantly higher than in those without complications $(p=0.037)$. The types of $\mathrm{CP}$ were not significantly different between the groups with and without complications $(p=0.785)$. The pre( $p=0.049)$ and postoperative JOA scores $(p=0.042)$ were significantly less in those with postoperative complications than in those without postoperative complications; however, the JOA recovery rate did not significantly differ $(p=0.892)$. No other clinical outcomes significantly differed between those with and without postoperative complications. The preoperative and postoperative radiologic parameters also did not differ significantly (Table 6). 
Table 5. Clinical outcomes according to the presence or absence of complications in the cerebral palsy group

\begin{tabular}{|c|c|c|c|}
\hline & YC $(n=16)$ & NC $(n=19)$ & $p$-value \\
\hline \multicolumn{4}{|l|}{ Clinical outcome } \\
\hline Pre-op JOA & $11.38 \pm 4.60$ & $13.95 \pm 2.76$ & $0.049^{*}$ \\
\hline Post-op JOA score & $13.50 \pm 3.67$ & $15.74 \pm 2.58$ & $0.042^{*}$ \\
\hline JOA recovery rate (\%)* & $25.30 \pm 21.07$ & $26.32 \pm 22.61$ & 0.892 \\
\hline Pre-op NDI & $39.50 \pm 20.64$ & $37.95 \pm 17.97$ & 0.813 \\
\hline Post-op NDI & $26.75 \pm 16.96$ & $28.05 \pm 16.71$ & 0.821 \\
\hline Pre-op MBI & $42.94 \pm 23.22$ & $57.53 \pm 24.89$ & 0.084 \\
\hline Post-op MBI & $62.19 \pm 19.49$ & $72.11 \pm 20.50$ & 0.154 \\
\hline \multicolumn{4}{|l|}{ Grip and pinch test (kg) } \\
\hline Pre-op grip & $18.01 \pm 9.41$ & $18.17 \pm 7.99$ & 0.956 \\
\hline Post-op grip & $15.06 \pm 9.23$ & $16.11 \pm 8.99$ & 0.736 \\
\hline Pre-op tip & $2.62 \pm 1.68$ & $2.74 \pm 1.78$ & 0.842 \\
\hline Post-op tip & $2.57 \pm 1.70$ & $2.22 \pm 1.47$ & 0.515 \\
\hline Pre-op lateral & $3.88 \pm 1.85$ & $4.15 \pm 2.18$ & 0.696 \\
\hline Post-op lateral & $3.79 \pm 1.99$ & $3.62 \pm 1.88$ & 0.795 \\
\hline Pre-op palmar & $3.42 \pm 1.66$ & $3.52 \pm 2.18$ & 0.888 \\
\hline Post-op palmar & $4.01 \pm 1.58$ & $3.68 \pm 2.56$ & 0.665 \\
\hline \multicolumn{4}{|l|}{ Box \& Block test } \\
\hline Pre-op & $26.38 \pm 16.09$ & $23.57 \pm 15.98$ & 0.734 \\
\hline Post-op & $23.50 \pm 12.89$ & $24.69 \pm 16.40$ & 0.689 \\
\hline \multicolumn{4}{|l|}{$\begin{array}{l}\text { Jebsen-Taylor hand function } \\
\text { test (seconds) }\end{array}$} \\
\hline Writing, pre-op & $54.67 \pm 48.68$ & $45.81 \pm 27.70$ & 0.505 \\
\hline Writing, post-op & $43.61 \pm 29.31$ & $39.36 \pm 25.81$ & 0.652 \\
\hline Card turning, pre-op & $19.10 \pm 12.30$ & $17.02 \pm 11.16$ & 0.604 \\
\hline Card turning, post-op & $14.28 \pm 8.42$ & $13.13 \pm 9.02$ & 0.701 \\
\hline Moving light objects, pre-op & $12.35 \pm 10.82$ & $12.74 \pm 11.83$ & 0.919 \\
\hline Moving light objects, post-op & $9.44 \pm 3.96$ & $9.24 \pm 6.55$ & 0.917 \\
\hline Moving heavy objects, pre-op & $9.90 \pm 7.37$ & $10.43 \pm 7.75$ & 0.839 \\
\hline Moving heavy objects, post-op & $9.40 \pm 7.06$ & $9.02 \pm 6.69$ & 0.873 \\
\hline Stacking checkers, pre-op & $22.09 \pm 14.37$ & $19.43 \pm 13.04$ & 0.575 \\
\hline Stacking checkers, post-op & $19.22 \pm 14.93$ & $15.85 \pm 13.80$ & 0.493 \\
\hline Simulated feeding, pre-op & $23.07 \pm 14.73$ & $27.14 \pm 18.72$ & 0.487 \\
\hline Simulated feeding, post-op & $23.29 \pm 16.75$ & $21.36 \pm 14.72$ & 0.719 \\
\hline Placing small objects, pre-op & $36.15 \pm 42.43$ & $24.85 \pm 18.50$ & 0.301 \\
\hline Placing small objects, post-op & $26.20 \pm 19.94$ & $18.46 \pm 11.15$ & 0.158 \\
\hline
\end{tabular}

Values are presented as mean \pm standard deviation. *JOA recovery rate (\%): [(preoperative JOA score - postoperative JOA score) / (21 - preoperative JOA score) $\times 100$. YC : yes complication, NC : no complication, Pre-op : preoperative, JOA : Japanese Orthopaedic Association, Post-op : postoperative, NDI : Neck disability index; MBI : modified Barthel index
Table 6. Radiologic outcomes according to the presence or absence of complications in the cerebral palsy group

\begin{tabular}{lrrr}
\hline & YC $(\mathbf{n}=16)$ & NC $(\mathbf{n}=19)$ & $p$-value \\
\hline Pre-op radiologic parameters & & & \\
C0-C2 angle, neutral & $43.76 \pm 20.33$ & $38.01 \pm 13.61$ & 0.326 \\
C2-C7 angle, neutral & $2.45 \pm 24.48$ & $5.77 \pm 24.45$ & 0.691 \\
C2-C7 angle, flexion & $-31.68 \pm 15.46$ & $-30.44 \pm 19.08$ & 0.836 \\
C2-C7 angle, extension & $19.41 \pm 22.37$ & $24.78 \pm 21.22$ & 0.472 \\
T1 slope & $28.15 \pm 13.56$ & $24.88 \pm 14.14$ & 0.493 \\
T1 slope-C27 angle & $25.70 \pm 18.21$ & $19.11 \pm 16.45$ & 0.269 \\
C2-7 SVA (cm) & $28.29 \pm 26.97$ & $24.18 \pm 19.01$ & 0.601 \\
C2-7 ROM & $51.09 \pm 29.12$ & $55.23 \pm 20.02$ & 0.624 \\
CBVA & $-3.93 \pm 14.00$ & $-0.08 \pm 17.81$ & 0.488 \\
Post-op radiologic parameters & & & \\
C0-C2 angle, neutral & $41.64 \pm 20.47$ & $39.93 \pm 19.77$ & 0.803 \\
C2-C7 angle, neutral & $-0.28 \pm 19.59$ & $4.36 \pm 26.34$ & 0.565 \\
C2-C7 angle, flexion & $-21.38 \pm 14.94$ & $-24.85 \pm 16.92$ & 0.527 \\
C2-C7 angle, extension & $11.21 \pm 17.82$ & $15.66 \pm 26.96$ & 0.577 \\
T1 slope & $24.70 \pm 12.74$ & $24.76 \pm 14.31$ & 0.989 \\
T1 slope-C27 angle & $24.97 \pm 18.49$ & $20.40 \pm 18.43$ & 0.471 \\
C2-7 SVA (cm) & $25.87 \pm 29.27$ & $25.44 \pm 19.38$ & 0.959 \\
C2-7 ROM & $32.59 \pm 25.17$ & $40.51 \pm 29.06$ & 0.400 \\
CBVA & $-0.93 \pm 13.55$ & $-1.53 \pm 10.23$ & 0.881 \\
\hline
\end{tabular}

Values are presented as mean \pm standard deviation. YC : yes complication, NC : no complication, Pre-op : preoperative, SVA : sagittal vertical axis, ROM : range of motion, CBVA : chin brow vertical angle, Post-op: postoperative

\section{DISCUSSION}

$\mathrm{CP}$ is a movement disorder caused by a non-progressive abnormality appearing in the perinatal period. Lack of control and/or spasticity of truncal muscles frequently lead to spinal degenerative diseases or deformities, such as CSM and scoliosis. Early degeneration onset and malalignment of the spine have been reported in patients with CSM and CP. Owing to repetitive and unusual movements associated with this condition, the pathophysiology of CSM accelerates in patients with $\mathrm{CP}$, and inexplicable changes or deterioration of neurological function requiring surgical treatments can occur ${ }^{19,21)}$. CSM surgery for patients with CP remains challenging because of perioperative instrumentation failure caused by the patient's repetitive involuntary neck movements, complicated cervical spine deformities, and comorbidities. Several studies have reported 
postoperative complications in patients with CSM without CP or scoliosis with $\mathrm{CP}^{24,33)}$. However, there exist only a few reports regarding postoperative complications in patients with CSM and CP. Current and accurate information regarding major complications following cervical surgeries for significant CSM in patients with CP is important for weighing the costs and benefits of corrective surgery for this population.

In previous studies, complication rates in NCP patients who underwent cervical surgery due to CSM were 9-30\% ${ }^{17,23,26,31)}$. Yaszay et al. ${ }^{33)}$ reported a rate of $36 \%$ for postoperative major complications (e.g., wound infections, pulmonary issues, instrument-related complications) in patients with CP with $>2$ years of follow-up after spinal surgery, with a spine-related reoperation rate of $14.0 \%$. Samdani et al. ${ }^{24)}$ reported a 39\% complication rate in 127 patients with CP who underwent spinal surgery. In our study, the overall postoperative complication rate for CSM surgery was significantly higher in patients with CP than in NCP patients. Additionally, there were significant group differences in terms of postoperative motor weakness, ASD, and revision events. There may be several reasons for these differences. Excessive involuntary neck motion induced by $\mathrm{CP}$, in association with vertebral slippage anteriorly or posteriorly after cervical fusion, could exaggerate segmental and kyphotic instability. Moreover, these involuntary movements, as well as the abnormal cervical spinal alignment due to an imbalance in cervical muscle tone and compression of neural elements by severe cervical spondylotic changes, can cause rapid and additional degenerative changes and subsequent neurological deficits ${ }^{12}$. In our study, six out of 35 patients in the CP group developed motor weakness. In three patients, motor weakness was caused by ASD, and in the other three patients, it was caused by progressive kyphosis. Some studies have reported that cervical ASD occurs in approximately 3\% of NCP patients. However, Azuma reported that ASD occurred in $30 \%$ of patients with CP with a follow-up period $>10$ years after cervical fusion for CSM. Likewise, in our study, ASD occurrence was significantly more common in the CP group than in the NCP group. However, instrument-related complications, such as pseudoarthrosis and screw fractures or loosening, did not significantly differ between CP and NCP groups. We hypothesize that improvement in surgical instrument and principles, leading to strong initial mechanical stability as a result of anterior plating or posterior screw fixation, contributed to these favorable results. Additionally, our results indicate that complications such as instrument failure or nonfusion may not be considered as strong as once thought when considering cervical surgery for CSM in patients with CP.

In our study, risk factors for postoperative complications in CSM with CP included fixed cervical deformity. This result was likely because among patients with $\mathrm{CP}$, surgical invasiveness, need for surgical release, and utilization of osteotomies significantly increased in those with fixed cervical kyphosis than in those with semi-rigid or flexible kyphosis. In patients with CP, fixed cervical kyphosis is often accompanied by severe degenerative changes; thus, surgical treatment of such patients is challenging and requires invasive surgery to correct and stabilize the deformity while decompressing neural elements and restoring sagittal alignment ${ }^{11,13,15)}$. Hence, patients with CSM and CP tend to undergo staged operations or combined approaches. Scheer et al. ${ }^{25)}$ reported significant differences in complication rates for different approaches (anterior approach, 27.3\%; posterior approach, 68.4\%; combined approach, $79.3 \%$ ). In the present study, many patients in the CP group required posterior or combined approaches (posterior with an anterior approach or anterior with a posterior approach), but there were no significant differences in surgical approaches between the CP and NCP groups. This is one of the reasons for the high postoperative complication rate.

Apart from the incidence of complications, the clinical outcome is another important factor used to assess the postoperative prognosis. Demura et al. ${ }^{3)}$ showed that the 10-year Barthel index and JOA score after cervical surgery significantly improved (by 36\% and 31\%, respectively) in 14 patients with CSM and CP. Additionally, Watanabe et al. ${ }^{29)}$ reported that the JOA score improved from 8.3 points preoperatively to 10.9 points by the final follow-up (mean, 58 months) in patients with cervical disorder-associated CP who underwent posterior fusion using cervical pedicle screws; however, one-third of the patients were unable to walk at the final follow-up owing to myelopathy progression. Other studies have shown that postoperative clinical outcomes for patients with cervical myelopathy and CP are relatively poor owing to unfavorable conditions (severe cervical spinal deformity and intervertebral instability associated with involuntary neck movements) ${ }^{2,32)}$. We compared patients with CSM and CP, according to the presence or absence of postoperative complications, to determine the trends in outcomes before and after surgery. We found that JOA scores before and after surgery significantly differed 
between those with and without complications; however, the JOA recovery rate, as well as NDI and MBI scores, did not significantly differ. Some studies have reported the imitations of JOA and Barthel index for the evaluation of patients with involuntary movements, as in $\mathrm{CP}^{4,9)}$. Therefore, additional indicators were needed for an accurate evaluation of this patient group $^{30)}$. Accordingly, we assessed the CP group using the functional indicators used in the field of rehabilitation medicine specializing in the evaluation of upper limb function for multilateral assessments (grip and pinch, Box and Block, and Jebsen-Taylor hand function tests). However, no significant differences were found between those with and without postoperative complications. Based on our results, it is not necessary to delay or cancel surgical treatments for patients with CSM and CP due to vague concerns of perioperative complication. Instead, careful consideration of the surgical methods and levels is required, and multidisciplinary consultations should be considered in the treatment of these patients.

Our study has a few key limitations. First, this was a retrospective, uncontrolled drawback study with small sample size. Moreover, the average follow-up period was longer in the CP group than in the NCP group, which was likely to cause selection bias in terms of the complication rate. Second, the rehabilitation medicine clinical scales were only applied to the CP group, and we did not conduct a multifaceted comparison of the clinical courses between the CP and NCP groups. Third, the instrumented level was included while matching the control group (NCP), but there was a fundamental difference in the surgical strategies between the CP and NCP groups. Additionally, the optimal prevention strategy for postoperative complications remains unclear; thus, additional studies are required. Third, the statistical analysis was limited, as this study was designed using a simple matching method for age, sex, and surgical range.

\section{CONCLUSION}

The incidence of overall complications, particularly ASD, postoperative motor weakness, and revision events, was higher in the CP group than in the NCP group during the follow-up period (at least 2 years). Risk factors for postoperative complications of CSM with CP included fixed cervical deformity. Instrument-related complications were not significantly different between groups. In the CP group, there were no differences in clinical outcomes between those with and without postoperative complications. These results suggest that postoperative complications may not be considered as a concern when deciding on surgical treatment for CSM in patients with CP.

\section{CONFLICTS OF INTEREST}

No potential conflict of interest relevant to this article was reported.

\section{INFORMED CONSENT}

This type of study does not require informed consent.

\section{AUTHOR CONTRIBUTIONS}

\author{
Conceptualization : HCK, YH, SRC \\ Data curation : HCK, JHH, SRC \\ Formal analysis : $\mathrm{HCK}, \mathrm{YH}, \mathrm{HJ}$ \\ Methodology : HCK, HJ, YHJ, SP, SBA \\ Project administration : HCK, YH, SRC \\ Visualization : HCK \\ Writing - original draft : HCK \\ Writing - review \& editing : HCK, DAS, SY, KNK
}

\section{ORCID}

Hyung Cheol Kim https://orcid.org/0000-0001-7147-9176

Hyeongseok Jeon https://orcid.org/0000-0002-8379-412X

Yeong Ha Jeong https://orcid.org/0000-0001-8150-2050

Sangman Park https://orcid.org/0000-0003-2768-2091

Seong Bae An https://orcid.org/0000-0002-3706-2075

Jeong Hyun Heo https://orcid.org/0000-0002-9734-5376

Dong Ah Shin https://orcid.org/0000-0002-5225-4083

Seong Yi https://orcid.org/0000-0003-0700-4744

Keung Nyun Kim https://orcid.org/0000-0003-2248-9188

Yoon Ha https://orcid.org/0000-0002-3775-2324

Sung-Rae Cho https://orcid.org/0000-0003-1429-2684 


\section{- Acknowledgements}

This research was supported by a grant of the Developing Regional Innovation Clusters R\&D Project through the Korea Institute for Advancement of Technology (KIAT), funded by Ministry of Trade, Industry and Energy, Republic of Korea (grant number: P0015342).

\section{References}

1. Anderson WW, Wise BL, Itabashi HH, Jones $\mathrm{M}$ : Cervical spondylosis in patients with athetosis. Neurology 12 : 410-412, 1962

2. Azuma S, Seichi A, Ohnishi I, Kawaguchi H, Kitagawa T, Nakamura $K$ : Long-term results of operative treatment for cervical spondylotic myelopathy in patients with athetoid cerebral palsy: an over 10-year follow-up study. Spine (Phila Pa 1976) 27 : 943-948; discussion 948, 2002

3. Demura S, Kato S, Shinmura K, Yokogawa N, Yonezawa N, Shimizu T, et al. : More than 10-year follow-up after laminoplasty and pedicle screw fixation for cervical myelopathy associated with athetoid cerebral palsy. Spine (Phila Pa 1976) 45 : 727-734, 2020

4. Demura S, Murakami H, Kawahara N, Kato S, Yoshioka K, Tsuchiya H : Laminoplasty and pedicle screw fixation for cervical myelopathy associated with athetoid cerebral palsy: minimum 5-year follow-up. Spine (Phila Pa 1976) 38 : 1764-1769, 2013

5. Fuji T, Yonenobu K, Fujiwara K, Yamashita K, Ebara S, Ono K, et al. : Cervical radiculopathy or myelopathy secondary to athetoid cerebral palsy. J Bone Joint Surg Am 69 : 815-821, 1987

6. Harada T, Ebara S, Anwar MM, Okawa A, Kajiura I, Hiroshima K : The cervical spine in athetoid cerebral palsy. A radiological study of 180 patients. J Bone Joint Surg Br 78 : 613-619, 1996

7. Hartmann S, Thomé C, Abramovic A, Lener S, Schmoelz W, Koller J, et al. : The effect of rod pattern, outrigger, and multiple screw-rod constructs for surgical stabilization of the 3-column destabilized cervical spine - a biomechanical analysis and introduction of a novel technique. Neurospine $17:$ 610-629, 2020

8. Hasler CC : Operative treatment for spinal deformities in cerebral palsy. J Child Orthop $7:$ 419-423, 2013

9. Jameson R, Rech C, Garreau de Loubresse C : Cervical myelopathy in athetoid and dystonic cerebral palsy: retrospective study and literature review. Eur Spine J 19 : 706-712, 2010

10. Kidron D, Steiner I, Melamed E : Late-onset progressive radiculomyelopathy in patients with cervical athetoid-dystonic cerebral palsy. Eur Neurol 27 : 164-166, 1987

11. Kim CW, Hyun SJ, Kim KJ : Surgical impact on global sagittal alignment and health-related quality of life following cervical kyphosis correction surgery: systematic review. Neurospine 17 : 497-504, 2020

12. Kim KN, Ahn PG, Ryu MJ, Shin DA, Yi S, Yoon DH, et al. : Long-term sur- gical outcomes of cervical myelopathy with athetoid cerebral palsy. Eur Spine J 23 : 1464-1471, 2014

13. Lau $D$, Ames CP : Three-column osteotomy for the treatment of rigid cervical deformity. Neurospine 17 : 525-533, 2020

14. Lee CK, Shin DA, Yi S, Kim KN, Shin HC, Yoon DH, et al. : Correlation between cervical spine sagittal alignment and clinical outcome after cervical laminoplasty for ossification of the posterior longitudinal ligament. J Neurosurg Spine 24 : 100-107, 2016

15. Lee JJ, Oh SH, Jeong YH, Park SM, Jeon HS, Kim HC, et al. : Surgical strategies for cervical deformities associated with neuromuscular disorders. Neurospine 17 : 513-524, 2020

16. Lee YJ, Chung DS, Kim JT, Bong HJ, Han YM, Park YS : Surgical treatments for cervical spondylotic myelopathy associated with athetoid cerebral palsy. J Korean Neurosurg Soc 43 : 294-299, 2008

17. Master DL, Son-Hing JP, Poe-Kochert C, Armstrong DG, Thompson GH : Risk factors for major complications after surgery for neuromuscular scoliosis. Spine (Phila Pa 1976) 36 : 564-571, 2011

18. McCluer $S$ : Cervical spondylosis with myelopathy as a complication of cerebral palsy. Paraplegia 20 : 308-312, 1982

19. Nishihara N, Tanabe G, Nakahara S, Imai T, Murakawa H : Surgical treatment of cervical spondylotic myelopathy complicating athetoid cerebral palsy. J Bone Joint Surg Br 66 : 504-508, 1984

20. Piazzolla A, Solarino G, De Giorgi S, Mori CM, Moretti L, De Giorgi G : Cotrel-Dubousset instrumentation in neuromuscular scoliosis. Eur Spine J 20 Suppl 1 : S75-S84, 2011

21. Polk JL, Maragos VA, Nicholas JJ : Cervical spondylotic myeloradiculopathy in dystonia. Arch Phys Med Rehabil 73 : 389-392, 1992

22. Pollard CA : Preliminary validity study of the pain disability index. Percept Mot Skills 59 : 974, 1984

23. Rumalla K, Yarbrough CK, Pugely AJ, Koester L, Dorward IG : Spinal fusion for pediatric neuromuscular scoliosis: national trends, complications, and in-hospital outcomes. J Neurosurg Spine 25 : 500-508, 2016

24. Samdani AF, Belin EJ, Bennett JT, Miyanji F, Pahys JM, Shah SA, et al. : Major perioperative complications after spine surgery in patients with cerebral palsy: assessment of risk factors. Eur Spine J 25 : 795-800, 2016

25. Scheer JK, Tang JA, Smith JS, Acosta FL Jr, Protopsaltis TS, Blondel B, et al. : Cervical spine alignment, sagittal deformity, and clinical implications: a review. J Neurosurg Spine 19 : 141-159, 2013

26. Sharma $S$, Wu C, Andersen $T$, Wang $Y$, Hansen ES, Bünger CE : Prevalence of complications in neuromuscular scoliosis surgery: a literature meta-analysis from the past 15 years. Eur Spine J 22 : 1230-1249, 2013

27. Shimokawa $\mathrm{N}$, Sato $\mathrm{H}$, Matsumoto $\mathrm{H}$, Takami T : Complex revision surgery for cervical deformity or implant failure. Neurospine 17 : 543553,2020

28. Vialle $R$, Thévenin-Lemoine $C$, Mary $P$ : Neuromuscular scoliosis. Orthop Traumatol Surg Res 99 : S124-S139, 2013

29. Watanabe K, Hirano T, Katsumi K, Ohashi M, Shoji H, Yamazaki A, et al. : Surgical outcomes of posterior spinal fusion alone using cervical 
pedicle screw constructs for cervical disorders associated with athetoid cerebral palsy. Spine (Phila Pa 1976) 42 : 1835-1843, 2017

30. Watanabe K, Otani K, Nikaido T, Kato K, Kobayashi H, Yabuki S, et al. : Surgical outcomes of cervical myelopathy in patients with athetoid cerebral palsy: a 5-year follow-up. Asian Spine J11 : 928-934, 2017

31. Wewel JT, Brahimaj BC, Kasliwal MK, Traynelis VC : Perioperative complications with multilevel anterior and posterior cervical decompression and fusion. J Neurosurg Spine 20 : 1-6, 2019

32. Wong AS, Massicotte EM, Fehlings MG : Surgical treatment of cervical myeloradiculopathy associated with movement disorders: indications, technique, and clinical outcome. J Spinal Disord Tech 18 Suppl : S107-S114, 2005

33. Yaszay B, Bartley CE, Sponseller PD, Abel M, Cahill PJ, Shah SA, et al. : Major complications following surgical correction of spine deformity in 257 patients with cerebral palsy. Spine Deform 8 : 1305-1312, 2020

34. Yonenobu K, Okada K, Fuji T, Fujiwara K, Yamashita K, Ono K : Causes of neurologic deterioration following surgical treatment of cervical myelopathy. Spine (Phila Pa 1976) 11 : 818-823, 1986 